\title{
Enyesado de suelos sódicos: modificación de sus propiedades químicas, físicas y biológicas
}

\section{Gypsum soil amendment: modification of its chemical, physical and biological properties}

\section{Esteban Melani}

INTA AER Chascomús, Argentina

\section{Facundo Guilino}

Centro de Investigación en Sustentabilidad de Suelos Agrícolas y Forestales (CISSAF), Facultad de Ciencias Agrarias y Forestales, UNLP, Argentina

\section{Laura Mónica Draghi}

Centro de Investigación en Sustentabilidad de Suelos Agrícolas y Forestales (CISSAF), Facultad de Ciencias Agrarias y Forestales, UNLP, Argentina

\section{Daniel Jorajuría}

Centro de Investigación en Sustentabilidad de Suelos Agrícolas y Forestales (CISSAF), Facultad de Ciencias Agrarias y Forestales, UNLP, Argentina

\section{Rafael Villarreal}

Centro de Investigación en Sustentabilidad de Suelos Agrícolas y Forestales (CISSAF), Facultad de Ciencias Agrarias y Forestales, UNLP; Consejo Nacional de Investigaciones Científicas y Técnicas (CONICET), Argentina

\section{Luis Lozano}

Centro de Investigación en Sustentabilidad de Suelos Agrícolas y Forestales (CISSAF), Facultad de Ciencias Agrarias y Forestales, UNLP; Consejo Nacional de Investigaciones Científicas y Técnicas (CONICET), Argentina

\section{Germán Soracco}

Centro de Investigación en Sustentabilidad de Suelos Agrícolas y Forestales (CISSAF), Facultad de Ciencias Agrarias y Forestales, UNLP; Consejo Nacional de Investigaciones Científicas y Técnicas (CONICET), Argentina

\section{Telmo Palancar *}

Centro de Investigación en Sustentabilidad de Suelos Agrícolas y Forestales (CISSAF), Facultad de Ciencias Agrarias y Forestales, UNLP, Argentina

\section{Revista de la Facultad de Agronomía}

Universidad Nacional de La Plata, Argentina

ISSN: 1669-9513

Periodicidad: Semestral

Vol. 120, núm. 2, 2021

redaccion.revista@agro.unlp.edu.ar

Recepción: 11/08/2020

Aprobación: 22/08/20

URL: http://portal.amelica.org/ameli/jatsRepo/23/232371008/index.html

DOI: https://doi.org/10.24215/16699513e079

*Autor de correspondencia: telmo@agro.unlp.edu.ar 


\title{
Resumen
}

Los suelos sódicos ocupan una extensa proporción de la Depresión del Salado. El sodio aumenta el $\mathrm{pH}$ y provoca la dispersión de las arcillas generando modificaciones en la estructura de los suelos que perjudican el crecimiento de los cultivos al reducir su porosidad y su infiltración. La aplicación de yeso puede mejorar las características de los suelos sódicos. Se realizó un ensayo en el que se aplicaron tres dosis de yeso: A) $0 \mathrm{~kg} / \mathrm{ha}$ (testigo); B) $100 \mathrm{~kg} / \mathrm{ha}$ (en la línea de siembra) y C) 12000 $\mathrm{kg} / \mathrm{ha}$ (en cobertura total). Se replicó el ensayo usando dos cultivos: Festuca (Festuca arundinacea Schreb.) y Agropiro (Thynopirum ponticum Barkworth \& D.R.Dewey). En los distintos tratamientos se midieron propiedades químicas $(\mathrm{pH}$, conductividad eléctrica, porcentaje de sodio intercambiable (PSI) y carbono orgánico), físicas (resistencia a la penetración, resistencia al corte, densidad aparente e infiltración) y biológicas (porcentaje de implantación y productividad primaria neta). El yeso redujo el pH, aumentó la conductividad eléctrica y redujo el PSI. Además, no modificó la resistencia al corte del suelo, pero sí aumentó su capacidad portante en una situación de elevada humedad y mejoró su conductividad hidráulica. Si bien todos estos cambios mejoraron el porcentaje de implantación de ambos cultivos no se modificó la producción de materia seca.

Palabras clave: infiltración, resistencia a la penetración, materia seca, porosidad, alcalinidad

\begin{abstract}
Sodic soils occupy a large proportion of the Salado Depression. Sodium increases the $\mathrm{pH}$ and causes the dispersion of the clays, generating modifications in the structure of soils that harm the growth of crops by reducing their porosity and infiltration. The application of gypsum can improve the characteristics of sodic soils. A test was carried out in which three doses of gypsum were applied: A) $0 \mathrm{~kg} / \mathrm{ha}$ (control); B) $100 \mathrm{~kg} / \mathrm{ha}$ (in the seeding line) and C) $12000 \mathrm{~kg} / \mathrm{ha}$ (in full coverage). The test was replicated using two crops: Fescue (Festuca arundinacea Schreb.) and Agropiro (Thynopirum ponticum Barkworth \& D.R.Dewey). In the different treatments, chemical ( $\mathrm{pH}$, electrical conductivity, exchangeable sodium percentage (ESP) and organic carbon), physical (penetration resistance, shear resistance, bulk density and infiltration) and biological (percentage of implantation and net primary productivity) were measured. Gypsum lowered $\mathrm{pH}$, increased electrical conductivity, and lowered ESP. In addition, it did not modify the shear resistance of the soil, but it did increase its load-bearing capacity in a situation of high humidity and improved its hydraulic conductivity. Although all these changes improved the implantation percentage of both crops, dry matter production was not modified.
\end{abstract}

Keywords: soil infiltration, soil penetration resistance, dry matter, porosity, soil alkalinity 


\section{INTRODUCCION}

Un $60 \%$ de la superficie de la Depresión del Salado está afectada por exceso de sodio. La alcalinización de los suelos conduce a un deterioro de sus propiedades químicas y físicas. Las características típicas de estos suelos son la acumulación de sales de sodio, un elevado $\mathrm{pH}$, una elevada relación de absorción de sodio (RAS) y un elevado porcentaje de sodio intercambiable (PSI) en la superficie (Qadir \& Schubert, 2002). Estos factores causan desestabilización de la estructura del suelo, deterioro de las propiedades hidráulicas y un desbalance de los nutrientes disponibles para las plantas resultando en una pobre cobertura vegetal. Adicionalmente, una estructura pobre del suelo conduce a una baja permeabilidad del agua (Chi \& Wang, 2010).

La dispersión de la arcilla origina el encostramiento y endurecimiento de la superficie del suelo. Esto incrementa la resistencia y disminuye la tasa de infiltración, la porosidad y la germinación (Barzegar et al., 1994). Wong et al. (2006) sostienen que los suelos sódicos se dispersan al humedecerse, formando estructuras endurecidas masivas cuando se secan relacionadas en gran medida a la disminución de la infiltración y formación de costras superficiales. Palancar et al. (2006) encontraron mayores resistencias al corte en suelos con mayores niveles de sodio. Otondo et al. (2015) utilizaron un penetrómetro Proctor para determinar la capacidad portante de suelos sódicos implantados con especies megatérmicas y encontraron una mejora en los mismos en sus propiedades físicas incluida su capacidad portante que aumentó.

Shainberg \& Letey (1984), Jayawardane \& Chan (1994), Lebron et al. (1994), demostraron que altos niveles de PSI causan hinchamiento y dispersión de las arcillas, ocasionando encostramiento, reducción de la infiltración, pérdida de estabilidad estructural y reducción en el crecimiento de las plantas debido a estas alteraciones y a la toxicidad específica del sodio.

Costa \& Aparicio (2000) encontraron que la conductividad hidráulica saturada se redujo exponencialmente con PSI mayores al $5 \%$. Es suficiente una pequeña cantidad de sodio adsorbido para aumentar la dispersión de la arcilla. Trabajos de Barzegar et al. (1994) han demostrado que un incremento del PSI produce un incremento de la arcilla dispersa y por lo tanto de la resistencia del suelo. La presencia de sodio influye negativamente en el laboreo de los suelos.

Varallyay (2002) afirma que un alto PSI ocasiona una significativa modificación de la estructura y más concretamente de la conformación del espacio poroso, modificando la compactabilidad de los suelos. Palancar et al. (2006) hallaron mayor susceptibilidad a la compresión en los suelos menos afectados por sodio debido probablemente a la presencia de poros de mayor tamaño. Una estructura de partículas floculadas presenta grandes poros mientras que una dispersa tiene numerosos microporos y ante tensiones elevadas el efecto de las tensiones es mayor sobre las estructuras floculadas (Mitchell, 1976). Ante un incremento dado de tensión, los poros de mayor tamaño son comprimidos primero (Delage \& Lefebvre, 1984; Griffiths \& Joshi, 1989). En el mismo sentido, Spugnoli \& Melani (2005) reportaron menos sensibilidad a la compresión para los suelos más afectados por sodio.

La aplicación de enmiendas químicas, como el yeso, es el método más difundido en el mundo para recuperar suelos sódicos. Costa \& Godz (1998) reportaron un incremento de la conductividad eléctrica (CE) a la profundidad que el yeso fue mezclado con el suelo. La CE disminuyó con el tiempo por la acción del lavado de sales provocado por las lluvias y por los ascensos freáticos, mientras que en períodos de sequía y descenso freático sufrió incrementos. Costa \& Godz (1999) comprobaron que el agregado de yeso produjo una mejora en un suelo Natracuol, favoreciendo la implantación de pasturas y duplicando la producción de materia seca. Mendoza \& Barbieris (1980) concluyeron que el agregado de yeso a un suelo natracualf indujo una evolución del suelo sódico a la condición de salino-sódico, disminuyendo su pH, RAS y aumentando su CE. También Costa \& Godz $(1998,1999)$ encontraron incrementos de CE y reducciones de RAS en suelos enyesados. Bandera (2013) evaluó la aplicación de yeso y diferentes enmiendas biológicas sobre un suelo Natracuol típico, encontrando mejoras en los parámetros químicos del suelo y un mayor crecimiento de Agropiro (Thynopirum ponticum Barkworth \& D.R.Dewey) bajo ensayos controlados, pero a campo no encontró efecto alguno sobre las propiedades químicas del suelo.

No son abundantes los trabajos realizados a campo en cuanto al desempeño del uso de enmiendas químicas en suelos halomórficos de la Depresión del Salado, sobre todo en lo referente a suelos Natracualf. En estos suelos sódicos la lixiviación del sodio está muy limitada por el horizonte Bt nátrico y la poca profundidad a la que se halla la napa freática impidiendo el lavado de las sales, razón por la cual los resultados encontrados en trabajos con muestras drenadas difícilmente se logren en ensayos de campo 0 se reviertan en un muy corto período de tiempo. La falta de resultados obtenidos por el uso de enmiendas químicas en suelos halomórficos de la Pampa Deprimida, sumado al hecho de que el uso de yeso implica 
la aplicación de altos volúmenes (10-60 Mg/ha de yeso) con una difícil logística y cuyo costo económico es elevado, es la causa por la cual su uso no se ha generalizado.

El objetivo del trabajo fue evaluar a campo la respuesta a la aplicación de dos dosis de yeso en la implantación y crecimiento de dos especies perennes Festuca (Festuca arundinacea Schreb.) y Agropiro en un suelo Natracualf, como también los cambios en parámetros químicos y físicos que sirvan para inferir una mejora de estos ambientes. El objetivo específico fue el de evaluar si la aplicación de una dosis baja de yeso en la línea de siembra, más ajustada a las posibilidades de los productores, genera algún efecto positivo en la productividad primaria de un suelo Natracualf.

\section{MATERIALES Y MÉTODOS}

Se realizó un ensayo en la Chacra Experimental Integrada Chascomús INTA-MAIBA, en las proximidades de las coordenadas $35^{\circ} 44^{\prime} 06,5^{\prime \prime} S$ y $58^{\circ} 03^{\prime} 11,2^{\prime \prime} \mathrm{O}$. El suelo utilizado fue un Natracualf típico fina, illítica, térmica de la Serie Los Mochos con una capacidad de uso VIws. La napa freática se encuentra a una profundidad media de 1,50 m (INTA, 2010) si bien, de forma empírica, se observa frecuentemente que puede llegar al límite inferior del horizonte Btna, cercano a los 0,70 m. El ensayo se llevó a cabo en parcelas totalmente aleatorizadas y constó de dos tratamientos con dos especies perennes: 1) Festuca y 2) Agropiro y a su vez se realizaron tres subtratamientos: A: $0 \mathrm{~kg} / \mathrm{ha}$ de yeso; B: $100 \mathrm{~kg} / \mathrm{ha}$ de yeso en la línea de siembra y C: $12.000 \mathrm{~kg} / \mathrm{ha}$ de yeso en cobertura total. Se replicó el ensayo en tres bloques. Cada subtratamiento se realizó en parcelas de $6 \mathrm{~m} \times 0,9 \mathrm{~m}$, totalizando 18 parcelas (2 tratamientos $\times 3$ subtratamientos $\times 3$ bloques). La dosis de yeso del subtratamiento $C$ se basó en el trabajo realizado por Mendoza \& Barbieris (1980) en un suelo similar y con el que hallaron diferencias en la productividad primaria neta (PPN) respecto al testigo sin tratar. Fue también similar a la menor tasa de aplicación de yeso (15 Mg/ha) utilizada por Costa \& Godz (1999) para enmendar un Natracuol.

La superficie destinada al ensayo fue primero pulverizada con glifosato en marzo de 2016 con el fin de eliminar el pastizal existente. Posteriormente se realizó el laboreo mediante rastra de discos (2 pasadas) y arado rotativo y se aplicó el yeso al voleo en las parcelas correspondientes al subtratamiento C para inmediatamente realizar una nueva pasada de arado rotativo a todo el ensayo con el fin de incorporar el yeso aplicado. El yeso utilizado (Yeso agrícola Roca Fértil) tuvo un contenido de sulfato de calcio del $90 \%$, $16,7 \%$ de azufre (S) (elemento) y $21 \%$ de calcio $\left(\mathrm{Ca}^{++}\right)$(elemento). En mayo de 2016 se realizó la siembra, con sembradora experimental de 6 surcos a 0,15 m entre surcos, de Festuca y Agropiro con una densidad de $40 \mathrm{~kg} / \mathrm{ha}$ para ambas especies.

Las variables evaluadas fueron:

1. Químicas: $\mathrm{pH}$ y CE de 0 a $5 \mathrm{~cm}$ de profundidad y de 0 a $15 \mathrm{~cm}$ de profundidad, $\mathrm{PSI}$ de 0 a $15 \mathrm{~cm}$ de profundidad. El pH y CE se determinaron en extracto 1/2,5 mientras que la determinación de la CIC se realizó mediante filtrado con acetato de amonio, después del cual sodio $\left(\mathrm{Na}^{+}\right)$y potasio $\left(\mathrm{K}^{+}\right)$se determinaron mediante espectrofotómetro de llama y calcio $\left(\mathrm{Ca}^{++}\right)$y magnesio $\left(\mathrm{Mg}^{++}\right)$mediante titulación con EDTA. A su vez se realizaron dos muestreos en diferentes momentos del ensayo, el primero en 2016 (inicial) junto con el conteo de plantas y el segundo en 2018 (final). También se determinó el contenido de carbono orgánico (CO). Para todas las determinaciones químicas se tomó una muestra compuesta en cada parcela totalizando tres muestras por subtratamiento.

2. Físicas: resistencia a la penetración (RP) mediante penetrómetro de cono electrónico (SC900 Soil Compaction Meter Fieldscout), construido bajo Norma ASAE EP542 (1999), con principio de medición de profundidad por ultrasonido. Los valores de índice de cono fueron agrupados en rangos de profundidad de $10 \mathrm{~cm}$ y se registraron hasta los $35 \mathrm{~cm}$ de profundidad. Se efectuaron 15 repeticiones por cada rango de profundidad y por cada tratamiento y subtratamiento. Densidad aparente con un cilindro de $307,7 \mathrm{~cm}^{3}$ de 0 a $10 \mathrm{~cm}$ de profundidad (Hillel, 1998). Se efectuaron 3 repeticiones por cada tratamiento y subtratamiento. Conductividad hidráulica a 0,3 y $6 \mathrm{~cm}$ de tensión (K0, K3 y K6) a partir de las tasas de infiltración básica obtenidas a campo mediante un infiltrómetro de disco a tensión (Perroux \& White, 1988), con un diámetro de disco de 12,5 cm, a través del método de cargas múltiples (Ankeny et al., 1991). Se realizaron 4 repeticiones en cada tratamiento y subtratamiento. Resistencia al corte (RC) en superficie mediante torquímetro de mano. Se efectuaron 5 repeticiones por tratamiento y subtratamiento. Humedad edáfica gravimétrica $\left(\mathrm{H}^{\circ}\right)$ en tres estratos $(0$ a $10 \mathrm{~cm}, 10$ a $20 \mathrm{~cm}$ y 20 a $30 \mathrm{~cm})$. La humedad gravimétrica se obtuvo por diferencia de peso de muestras de suelo húmedo y seco. El secado se realizó en estufa a $105^{\circ} \mathrm{C}$ 
durante $24 \mathrm{~h}$ (hasta peso constante). Se realizaron 3 repeticiones para cada tratamiento, subtratamiento y profundidad.

3. Biológicas: porcentaje de Implantación (Im) conociendo la cantidad de semillas sembradas por metro y contando las plantas emergidas en $1 \mathrm{~m}$ de surco en los 3 surcos centrales y la producción de materia seca (MS) en kg/ha de MS cortando para ello el material por encima de los $5 \mathrm{~cm}$ de altura en los 3 surcos centrales a lo largo de $6 \mathrm{~m}$ de surco y secándolo en estufa hasta peso constante (los cortes se realizaron en noviembre de 2016, enero, julio y noviembre de 2017 y en mayo de 2018 y de 2019).

Para todas las variables evaluadas se realizó un análisis de la varianza y se efectuó la comparación de medias mediante el test de diferencias mínimas significativas (LSD) de Fisher con un nivel de significancia del $5 \%$.

\section{RESULTADOS}

En la Figura 1 se observan las precipitaciones $(\mathrm{mm})$ ocurridas durante el período del ensayo en la Chacra Experimental Integrada Chascomús y el promedio histórico (1977-2019).

En la Tabla 1 se vuelcan el pH y la CE en los primeros $5 \mathrm{~cm}$ y en los primeros $15 \mathrm{~cm}$ y el PSI en los 15 $\mathrm{cm}$ superiores del suelo para ambos tratamientos determinados en 2016, junto con el conteo de plantas (determinación inicial).

En la Tabla 2 se vuelcan los mismos parámetros mostrados en la Tabla 1 pero determinados a mediados de 2018 (determinación final), dos años después de aplicado el yeso.

En la Tabla 3 se observan los valores de resistencia al corte $(R C)$ del suelo determinados con un torquímetro de mano.

En la Tabla 4 se muestran los valores de resistencia a la penetración (RP) registrados en ambos tratamientos para las distintas profundidades exploradas.

En la Tabla 5 se explicitan los valores de humedad gravimétrica en los tres estratos explorados mediante penetrometría (Tabla 4).

En la Tabla 6 se observan los valores de conductividad hidráulica, densidad aparente y carbono orgánico para cada tratamiento.

En la Tabla 7 se observa el porcentaje de implantación y la producción de MS para ambos tratamientos.

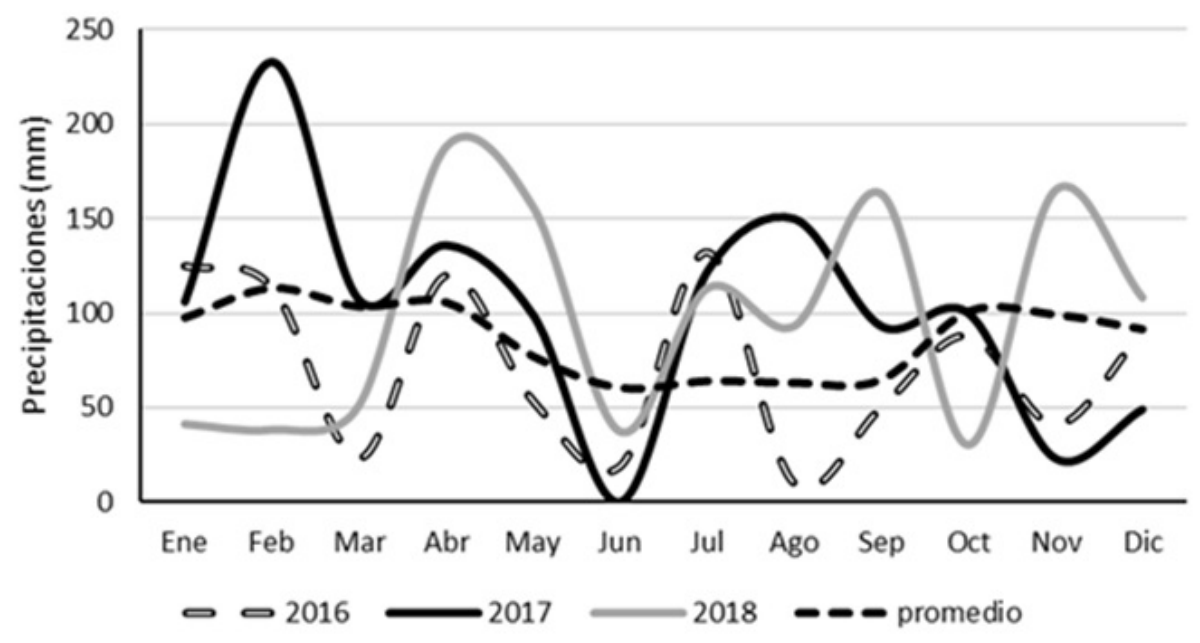

Figura 1

Precipitaciones registradas en la CEI Chascomús promedio del período 1977-2019 y para los años 2016, 2017 y 2018. 


\section{Tabla 1}

$\mathrm{pH}(\mathrm{pHi})$ y conductividad eléctrica (CEi, $\mathrm{dS} / \mathrm{m}$ ) en el estrato $0-5 \mathrm{~cm}$ y $0-15 \mathrm{~cm}$ y porcentaje de sodio intercambiable (PSli) iniciales en el estrato $0-15 \mathrm{~cm}$ para ambos tratamientos.

\begin{tabular}{cccccc}
\hline \multirow{2}{*}{ Subtratamiento } & $\mathrm{pHi}(\mathbf{0 - 5} \mathrm{cm})$ & $\mathrm{CEi}(0-5 \mathrm{~cm})$ & $\mathrm{pHi}(0-15 \mathrm{~cm})$ & $\mathrm{CEi}(0-15 \mathrm{~cm})$ & $\mathrm{PSli}(\mathbf{0 - 1 5} \mathrm{cm})$ \\
\cline { 2 - 6 } & $\mathrm{p}:<0,0001$ & $\mathrm{p}:<0,0001$ & $\mathrm{p}:<0,0001$ & $\mathrm{p}:<0,0001$ & $\mathrm{p}: 0,0027$ \\
$\mathrm{~A}(0)$ & $8,12 \mathrm{~b}$ & $0,34 \mathrm{a}$ & $8,65 \mathrm{~b}$ & $0,59^{\mathrm{a}}$ & $23,11 \mathrm{~b}$ \\
$\mathrm{~B}(100)$ & $8,09 \mathrm{~b}$ & $0,31 \mathrm{a}$ & $8,78 \mathrm{~b}$ & $0,55^{\mathrm{a}}$ & $24,32 \mathrm{~b}$ \\
$\mathrm{C}(12000)$ & $7,15 \mathrm{a}$ & $0,98 \mathrm{~b}$ & $7,91 \mathrm{a}$ & $1,09 \mathrm{~b}$ & $16,63 \mathrm{a}$ \\
\hline
\end{tabular}

Letras diferentes en la misma columna denotan diferencias mínimas significativas entre tratamientos mediante el Test de LSD $(p<0,05)$.

\section{Tabla 2}

$\mathrm{pH}(\mathrm{pHf})$, conductividad eléctrica (CEf, $d S / \mathrm{m}$ ) en el estrato $0-5 \mathrm{~cm}$ y $0-15 \mathrm{~cm}$ y porcentaje de sodio intercambiable (PSIf) finales en el estrato $0-15 \mathrm{~cm}$ para ambos tratamientos.

\begin{tabular}{cccccc}
\hline \multirow{2}{*}{ Subtratamiento } & pHf $(0-5 \mathrm{~cm})$ & CEf $(0-5 \mathrm{~cm})$ & pHf $(0-15 \mathrm{~cm})$ & CEf $(0-15 \mathrm{~cm})$ & PSIf $(0-15 \mathrm{~cm})$ \\
\cline { 2 - 6 } & p: 0,038 & p: 0,1882 & p: 0,0033 & p: 0,0200 & p: 0,0028 \\
A $(0)$ & $8,36 \mathrm{~b}$ & $0,46 \mathrm{a}$ & $8,20 \mathrm{~b}$ & $1,16 \mathrm{ab}$ & $28,26 \mathrm{~b}$ \\
B $(100)$ & $8,30 \mathrm{~b}$ & $0,49 \mathrm{a}$ & $8,23 \mathrm{~b}$ & $1,29 \mathrm{~b}$ & $28,85 \mathrm{~b}$ \\
C $(12000)$ & $7,95 \mathrm{a}$ & $0,39 \mathrm{a}$ & $7,80 \mathrm{a}$ & $0,98^{\mathrm{a}}$ & $19,06 \mathrm{a}$ \\
\hline
\end{tabular}

Letras diferentes en la misma columna denotan diferencias mínimas significativas entre tratamientos mediante el Test de LSD $(p<0,05)$.

Tabla 3

Resistencia al corte $(R C)$ de los tratamientos y subtratamientos.

\begin{tabular}{ccc}
\hline Subtratamiento & $\begin{array}{c}\text { RC Agropiro } \\
\left(\mathbf{k g} / \mathbf{c m}^{2}\right)\end{array}$ & $\begin{array}{c}\text { RC Festuca } \\
\left(\mathbf{k g} / \mathbf{c m}^{2}\right)\end{array}$ \\
\hline A $(0)$ & $2,30 a$ & $3,45 a$ \\
B (100) & $2,60 a$ & $2,80 a$ \\
C (12000) & $2,70 a$ & $3,00 a$ \\
\hline
\end{tabular}

Letras diferentes en la misma columna denotan diferencias mínimas significativas entre tratamientos mediante el Test de LSD $(p<0,05)$. 
Tabla 4

Resistencia a la penetración $(\mathrm{kPa})$ de los tratamientos y subtratamientos en los distintos estratos evaluados.

\begin{tabular}{ccccc}
\hline & \multicolumn{4}{c}{ Resistencia a la penetración $\mathbf{( k P a )}$} \\
Agropiro & $\mathbf{0 - 1 0} \mathbf{~ c m}$ & $\mathbf{1 0 - 2 0} \mathbf{~ c m}$ & $\mathbf{2 0 - 3 0} \mathbf{~ c m}$ & $\mathbf{3 0 - 3 5} \mathbf{~ c m}$ \\
\hline A (0) & $488,08 \mathrm{a}$ & $929,08 \mathrm{ab}$ & $817,4 \mathrm{c}$ & $1035,92^{\mathrm{a}}$ \\
$\mathrm{B}(100)$ & $547,68 \mathrm{a}$ & $1018,68 \mathrm{~b}$ & $738 \mathrm{~b}$ & $936,44 \mathrm{a}$ \\
C (12000) & $595,68 \mathrm{a}$ & $887,12 \mathrm{a}$ & $654,48 \mathrm{a}$ & $949,68 \mathrm{a}$ \\
Festuca & $\mathbf{0 - 1 0} \mathbf{~ c m}$ & $\mathbf{1 0 - 2 0} \mathbf{~ c m}$ & $\mathbf{2 0 - 3 0} \mathbf{~ c m}$ & $\mathbf{3 0 - 3 5} \mathbf{~ c m}$ \\
A (0) & $372,68 \mathrm{a}$ & $626,68 \mathrm{a}$ & $665,48 \mathrm{a}$ & $987,92 \mathrm{~b}$ \\
B (100) & $519,48 \mathrm{~b}$ & $788,52 \mathrm{~b}$ & $660,92 \mathrm{a}$ & $830,68^{\mathrm{a}}$ \\
C (12000) & $597,48 \mathrm{~b}$ & $971,6 \mathrm{c}$ & $734,28 \mathrm{~b}$ & $1003,92 \mathrm{~b}$ \\
\hline
\end{tabular}

Letras diferentes en la misma columna denotan diferencias mínimas significativas entre tratamientos mediante el Test de LSD $(p<0,05)$.

\section{Tabla 5}

Humedad gravimétrica (\%) relevada en los distintos estratos para ambos tratamientos.

\begin{tabular}{cccc}
\hline $\begin{array}{c}\text { Subtratamiento } \\
\text { (kg/ha) }\end{array}$ & $\mathbf{0 - 1 0} \mathbf{~ c m}$ & $\mathbf{1 0 - 2 0} \mathbf{~ m}$ & $\mathbf{2 0 - 3 0} \mathbf{~ c m}$ \\
A (0) & $34,85^{\mathrm{a}}$ & $33,80 \mathrm{a}$ & $44,13 \mathrm{a}$ \\
B (100) & $36,20^{\mathrm{a}}$ & $37,18 \mathrm{a}$ & $41,00 \mathrm{a}$ \\
C (12000) & $32,85^{\mathrm{a}}$ & $32,58 \mathrm{a}$ & $40,90 \mathrm{a}$ \\
\hline
\end{tabular}

Letras diferentes en la misma columna denotan diferencias mínimas significativas entre tratamientos mediante el Test de LSD $(p<0,05)$.

\section{Tabla 6}

Conductividad hidráulica a 0,3 y $6 \mathrm{~cm}$ de tensión (KO, K3 y K6), densidad aparente (DAP) y carbono orgánico (CO) de los tratamientos y subtratamientos evaluados.

\begin{tabular}{|c|c|c|c|c|c|c|}
\hline Tratamiento & Subtrat. & $\begin{array}{c}\text { K0 } \\
\text { (cm/hora) }\end{array}$ & $\begin{array}{c}\mathrm{K} 3 \\
\text { (cm/hora) }\end{array}$ & $\begin{array}{c}\text { K6 } \\
\text { (cm/hora) }\end{array}$ & $\begin{array}{c}\text { DAP } \\
(\mathrm{g} / \mathrm{cm} 3)\end{array}$ & $\begin{array}{c}\text { CO } \\
(\mathrm{g} / \mathrm{kg})\end{array}$ \\
\hline \multirow{3}{*}{ Agropiro } & $A(0)$ & $0,76^{a}$ & $0,59^{a}$ & $0,36^{a}$ & $1,15 a$ & $0,186 a$ \\
\hline & $B(100)$ & $2,22 \mathrm{~b}$ & $0,60^{a}$ & $0,20^{a}$ & $1,15 a$ & $0,199 a$ \\
\hline & $C(12000)$ & $1,67 a b$ & $0,67^{a}$ & $0,37^{a}$ & $1,17 a$ & $0,172 a$ \\
\hline Tratamiento & Subtrat. & $\begin{array}{c}\text { K0 } \\
\text { (cm/hora) }\end{array}$ & $\begin{array}{c}\mathrm{K} 3 \\
\text { (cm/hora) }\end{array}$ & $\begin{array}{c}\text { K6 } \\
\text { (cm/hora) }\end{array}$ & $\begin{array}{c}\text { DAP } \\
(\mathrm{g} / \mathrm{cm} 3)\end{array}$ & $\begin{array}{c}\mathrm{CO} \\
(\mathrm{g} / \mathrm{kg})\end{array}$ \\
\hline \multirow{3}{*}{ Festuca } & $A(0)$ & $2,23 a$ & $0,66 a b$ & $0,33 b$ & $1,11 \mathrm{a}$ & $0,161 b$ \\
\hline & B (100) & $2,10 a$ & $0,40^{\mathrm{a}}$ & $0,03^{a}$ & $1,10 a$ & $0,201 c$ \\
\hline & $C(12000)$ & $3,08 a$ & $0,98 b$ & $0,53 b$ & $1,12 a$ & $0,109 a$ \\
\hline
\end{tabular}

Letras diferentes en la misma columna denotan diferencias mínimas significativas entre tratamientos mediante el Test de LSD $(p<0,05)$. 
Tabla 7

Porcentaje de implantación y producción de materia seca para ambos tratamientos.

\begin{tabular}{|c|c|c|}
\hline Subtratamint & Implantación (\%) & MS (kg/ha) \\
\hline & p: 0,0141 & p: 0,6452 \\
\hline$A(0)$ & $21,85 a$ & $5135 a$ \\
\hline$B(100)$ & $26,63 a b$ & 4898a \\
\hline$C(12000)$ & $32,93 b$ & $5248 a$ \\
\hline
\end{tabular}

Letras diferentes en la misma columna denotan diferencias mínimas significativas entre tratamientos mediante el Test de LSD $(p<0,05)$.

\section{DISCUSIÓN}

Se observa que la dosis mayor de yeso ha reducido el $\mathrm{pH}$ y el PSI tanto inicial como final y ha aumentado la CE inicial (Tabla 1 y Tabla 2). Costa \& Godz (1998) reportan un incremento de la CE a la profundidad que el yeso fue mezclado con el suelo siendo el incremento proporcional al valor inicial de RAS. La CE final se ha reducido quizás por el lavado de las sales sobre todo de los años 2017 y 2018 más húmedos que la media histórica con una pluviometría acumulada entre el período inicial y final de $2100 \mathrm{~mm}$ (Figura 1). Además, en períodos húmedos puede elevarse el nivel de la napa freática y con esto diluirse las sales como encontraron Costa \& Godz (1999). La pérdida de CE del subtratamiento de $12000 \mathrm{~kg} / \mathrm{ha}$ de yeso podría haberse favorecido también por un aumento en su conductividad hidráulica provocado por una mejora en su estructura porosa. Más allá de perder CE el subtratamiento C mantiene en la determinación final un menor $\mathrm{pH}$ y PSI que los restantes subtratamientos. El subtratamiento de menor dosis de yeso (Subtratamiento B) no se ha diferenciado estadísticamente en ningún parámetro químico del subtratamiento control.

Los datos de reducción de $\mathrm{pH}$ en el estrato $0-15 \mathrm{~cm}$ a partir de la incorporación de yeso serían coincidentes con los obtenidos por Mendoza \& Barberis (1980) que encontraron una reducción en el pH, RAS y PSI y un aumento de la CE de suelos tratados con yeso. También Costa \& Godz (1998 y 1999) encontraron incrementos de CE y reducciones de RAS en suelos enyesados.

En resistencia al corte no se han encontrado diferencias entre subtratamientos (Tabla 3). Esta falta de variabilidad en la RC no coincidiría con lo encontrado por Barzegar et al. (1994), Palancar et al. (2006) y Wong et al. (2006) quienes manifestaron mayores impedancias en los suelos en mayor medida afectados por sodio. Al analizar diferencias entre los tratamientos (Agropiro vs Festuca) se encontraron mayores resistencia al corte en Festuca debido probablemente a las características radicales de cada cultivo.

En el parámetro resistencia a la penetración, se encontró una mayor impedancia en los subtratamientos con yeso en los horizontes superficiales (0-10 y 10-20) mientras que en los más profundos el subtratamiento sin yeso tuvo mayor impedancia (Tabla 4). En los subtratamientos con agregado e incorporación de yeso superficial podría haberse desplazado el sodio a los estratos inferiores y esto explicaría el comportamiento inverso del encontrado en el horizonte superficial respecto al subtratamiento control. La mayor impedancia superficial en los subtratamientos enmendados con yeso no coincide con lo encontrado por Barzegar et al. (1994) en cuanto a una mayor resistencia de los suelos sódicos o a la formación de sustancias endurecidas cuando secas como sostienen Wong et al. (2006). La humedad media al momento de la medición rondó el $37 \%$ (Tabla 5), cercano a capacidad de campo, lo que se corresponde con los bajos valores de resistencia a la penetración hallados, en general por debajo de los $1000 \mathrm{kPa}$. En esta condición límite de tan alta humedad una mayor resistencia a la penetración sería un indicador de una mejor capacidad portante, sinónimo de una mejor estructura.

Otondo et al. (2015) encontraron una menor capacidad portante de suelos sódicos como consecuencia de la utilización de especies megatérmicas. Atribuyeron este comportamiento a una reducción en la densidad aparente de los tratamientos con estas especies, que habrían aflojado el suelo causando un mejor 
ambiente para el enraizamiento durante el verano. En el presente ensayo no hubo diferencias de densidad aparente entre los subtratamientos que pudieran explicar diferencias en la resistencia a la penetración.

Tampoco se observaron diferencias significativas entre subtratamientos en la humedad gravimétrica que pudieran explicar las diferencias registradas en la impedancia mecánica (Tabla 5).

En el parámetro densidad aparente (Tabla 6) se observa un valor ligeramente mayor (no significativo) en el subtratamiento de mayor dosis de yeso. Esto podría ser explicado por la mayor compresibilidad de estos subtratamientos que tendrían una mayor cantidad de macroporos más fácilmente compresibles en coincidencia con lo manifestado por Delage \& Lefebvre (1984), Griffiths \& Joshi (1989), Varallyay (2002), Spugnoli \& Melani (2005) y Palancar et al. (2006).

La mayor infiltración observada en los subtratamientos con yeso también coincidiría en confirmar la existencia de poros de mayor tamaño en los mismos como lo afirman Mitchell (1976), Barzegar et al., (1994) y Varallyay (2002). La menor cantidad de sodio encontrada en estos subtratamientos explicaría la mejora en su conductividad hidráulica (Shainberg \& Letey, 1984; Barzegar et al., 1994; Jayawardane \& Chan,1994; Lebron et al., 1994; Costa \& Aparicio, 2000; Wong et al., 2006; Chi \& Wang, 2010).

La producción de MS fue significativamente mayor en Agropiro respecto a Festuca, quizás por una mejor adaptación genética de la especie para producir en estos ambientes. No se observan diferencias en la producción de MS entre subtratamientos (Tabla 7) pero sí se encontraron diferencias significativas en el porcentaje de implantación observándose una mejora con el aumento de la dosis de yeso. Estos resultados coinciden con lo encontrado por Costa \& Godz (1999) en cuanto a la implantación, pero no así en lo que hace a producción de MS reportado tanto por estos autores como por Bandera (2013). Las dosis aplicadas por Costa \& Godz (1999) fueron mayores a las utilizadas en el presente trabajo (de 15 a $60 \mathrm{Mg} / \mathrm{ha}$ ).

A pesar de no observarse una clara tendencia en cuanto a la producción de MS, se observa una incidencia positiva del agregado de yeso en las propiedades físicas de los suelos, en concordancia con Otondo et al. (2015). La alternativa de la incorporación de yeso en la línea, si bien no resulta tan efectiva en los cambios provocados en los suelos, es técnica y económicamente más viable. Resulta interesante poder evaluar en ensayos de mayor duración la evolución de dichas propiedades físicas y su impacto en la producción de forraje en distintas especies adaptadas.

\section{CONCLUSIONES}

Si bien la aplicación de yeso provocó mejoras en lo que respecta a la implantación e indicadores edáficos físicos y químicos como la disminución del PSI, el aumento de la infiltración y la capacidad portante, estos cambios no fueron suficientes para lograr una mayor producción forrajera de estos suelos. Posiblemente la degradación física de estos ambientes es tal que difícilmente se logren visualizar cambios productivos en el corto plazo. En consideración al objetivo específico de este trabajo, no se evidenció que la aplicación de yeso en la línea de siembra en una dosis económica y sencilla desde el punto de vista logístico haya logrado efectos productivos positivos, más allá de una tendencia en cuanto a la mejora en la implantación.

\section{BIBLIOGRAFIA}

Ankeny, M.D., M. Ahmed; T.C. Kaspar \& R. Horton. 1991. Simple field method for determining unsaturated hydraulic conductivity. Soil Science Society of America Journal 55: 467-470.

ASAE. 1999. Procedures for Using and Reporting Data Obtained with the Soil Cone Penetrometer, no. ASAE EP542, Inst. ASAE. St. Joseph, Michligan, USA.

Bandera, R. 2013. Rehabilitación de suelos salino-sódicos: evaluación de enmiendas y de especies forrajeras. Tesis de maestría. Escuela para Graduados Ing. Agr. Alberto Soriano, Facultad de Agronomía, Universidad de Buenos Aires. 56 pp. Disponible en: http://ri.agro.uba.ar/files/download/tesis/maestria/2013banderaramiro.pdf. Último acceso: Agosto de 2020.

Barzegar, A.R., G.J. Murray, G.J. Churchman \& P. Rengasamy. 1994. The strength of remoulded soils as affected by exchangeable cations and dispersible clay. Australian Journal of Soil Research 32: 185199. 
Chi, C.M. \& Z.C. Wang. 2010. Characterizing salt-a $\square$ ected soils of Songnen Plain using saturated paste and 1:5 soil-to-water extraction methods. Arid Land Research and Management 24: 1-11.

Costa, J.L. \& V. Aparicio. 2000. Efecto del sodio en la degradación de los suelos del sudeste de la Provincia de Buenos Aires, Argentina. 11a Conferencia de la Organización Internacional de la Conservación del Suelo. Buenos Aires. Argentina. pp. 15-16.

Costa, J.L. \& P. Godz. 1998. The effects of gypsum applied to a natraquoll of the Flooding Pampas of Argentina. Soil Use and Management 14: 246-247.

Costa, J.L. \& P. Godz. 1999. Aplicación de yeso a un natracuol del sudeste de la pampa deprimida. Ciencia del Suelo 17: 21-27.

Delage, P. \& G. Lefebvre. 1984. Study of the structure of a sensitive Champlain clay and of its evolution during consolidation. Canadian Geotechnical Journal 21:21-35.

Griffiths, F.J. \& R.C. Joshi. 1989. Change in pore size distribution due to consolidation of clays. Geotechnique 39: 159-67.

Hillel, D. 1998. Environmental Soil Physics. Fundamentals, Applications, and Environmental Considerations. Academic Press. 771 pp.

Instituto Nacional de Tecnología Agropecuaria. INTA. 2010. Carta de suelos de la República Argentina. hoja 3557-25 - Chascomús. http://anterior.inta.gov.ar/suelos/cartas/3557/Chascomus/index.htm Último acceso: Agosto de 2020.

Jayawardane, N.S. \& K.Y. Chan. 1994. The management of soil physical properties limiting crop production in Australian sodic soils. A review. Australian Journal of Soil Research 32: 13-44.

Lebron, I.; D.L. Suarez \& F. Alberto. 1994. Stability of the Calcareus Saline-Sodic Soil During Reclamation. Soil Science Society of America Journal 58: 1753-1762.

Mendoza R.E. \& L.A. Barberis. 1980. Efecto del agregado de yeso y el lavado a un suelo sódico de la depresión del Río Salado y su repercusión sobre la producción de Lolium perenne L. Revista de Investigaciones Agropecuarias 15: 297-304.

Mitchell, J.K. 1976. Fundamentals of Soil Behaviour. Wiley, New York. pp. 222-223.

Otondo, J., E.J. Jacobo \& M.A. Taboada. 2015. Mejora de propiedades físicas por el uso de especies megatérmicas en un suelo sódico templado. Ciencia del Suelo 33: 119-130.

Palancar, T., L. Draghi, M. Vázquez, P. Spugnoli \& E. Soverini. 2006. Resistencia al corte de suelos salinizados y sodificados por riego artificial. Resumen en Actas e In extenso en CD-Rom del XX Congreso Argentino de la Ciencia del Suelo. 1ra Reunión de Suelos de la Región Andina. Salta y Jujuy. Argentina.

Perroux, K.M. \& I. White. 1988. Design for disc permeameters. Soil Science Society of America Journal 52: $1205-1215$.

Qadir, M. \& S. Schubert. 2002. Degradation processes and nutrient constraints in sodic soils. Land Degradation and Development 13: 275-294.

Shainberg, I. \& J. Letey. 1984. Response of soils to sodic and saline condition. Hilgardia 52: 1-57.

Spugnoli, P. \& E. Melani. 2005. Proprietà meccaniche degli aggregati di un suolo sodicizzato. AllA2005. Catania. L'ingegneria agraria per lo sviluppo sostenibile dell'area mediterránea. Codice Lavoro: 1006.

Varallyay, G. 2002. Enviromental stresses induced by salinity/alcalinity in the Carpathian Basin (Central Europe). Symposium № 33, Paper № 1570. 17th World Congress of Soil Sciences. Tailandia.

Wong, V.N.L., R.S.B. Greene, R.C. Dalal, B.W. Murphy \& S. Mann. 2006. Carbon dynamics in saltaffected soils. 18th World Congress of Soil Science. Philadelphia, USA. 